Z Rheumatol 2010 $69: 770-771$ DOI 10.1007/s00393-010-0708-z

Online publiziert: 14. Oktober 2010

(c) Springer-Verlag 2010

\author{
J. Braun ${ }^{1}$ E. Genth ${ }^{2}$ · für den Vorstand der DGRh \\ ${ }^{1}$ Rheumazentrum Ruhrgebiet, Herne \\ ${ }^{2}$ Rheumaklinik und Rheumaforschungsinstitut, Aachen
}

\title{
Gegen das Vergessen
}

\author{
Stellungnahme der Deutschen Gesellschaft \\ für Rheumatologie (DGRh) zu den \\ nationalsozialistischen Verbrechen \\ an jüdischen Kollegen
}

Über die Geschichte der Rheumatologie in Deutschland während der nationalsozialistischen Herrschaft ist bisher wenig in der Öffentlichkeit bekannt. In der Diskussion der letzten Jahre um die mögliche Verstrickung deutscher Rheumatologen mit dem Nationalsozialismus wurden vor allem die Personen Hans Reiter [1] und Friedrich Wegener [2] genannt, deren Namen durch die bekannte Sonderform der reaktiven Arthritis („Reiter-Syndrom“) und die mit granulomatösen Veränderungen einhergehende Vaskulitis („Wegener-Granulomato$\mathrm{se}^{\text {") }} \mathrm{zu}$ international gebräuchlichen Eponymen geführt haben. Weniger bekannt ist, dass führende jüdische Mitglieder aus der Rheumatologie und Balneologie unter der nationalsozialistischen Verfolgung das Land verlassen mussten oder umgebracht wurden [3].

Der Vorstand der Deutschen Gesellschaft für Rheumatologie (DGRh) veröffentlicht diese Stellungnahme, um 65 Jahre nach dem Ende der nationalsozialistischen Verbrechensherrschaft ein Signal gegen das Vergessen dieses dunklen Teils der eigenen Geschichte zu setzen.

Die DGRh betrachtet die nationalsozialistischen Verbrechen mit Abscheu, Entsetzen und Beschämung. Die Tatsache, dass auch Ärzte und andere Kollegen, welche Bezüge zur Rheumatologie aufweisen, Mitschuld daran tragen, darf niemals in Vergessenheit geraten. Vielmehr müssen wir uns immer wieder daran erinnern, wie in Zeiten politischer Diktaturen und Indoktrinationen, aber auch ökonomischer Zwänge, schöpferische Leistungen mit schweren persönlichen Verstrickungen und Schuld verknüpft sein können oder, wie es Zaller [4] formuliert hat, „was geschehen kann, wenn es Ärzten nicht mehr bewusst ist, dass alle Menschen gleich erschaffen sind“.

Es ist der DGRh deshalb ein besonderes Anliegen, sich an die rassisch und politisch Verfolgten zu erinnern, die unter der Naziherrschaft ihre akademische Karriere unterbrechen, ihr Land verlassen und ihre Gesundheit und sogar ihr Leben opfern mussten. Neben den vielen nicht mehr namentlich Bekannten sind dies jüdische Mitglieder der Gesellschaft für Rheumabekämpfung wie Prof. Dr. Hermann Strauß (1864-1944), ein Gründungsmitglied unserer Fachgesellschaft [1], der im Konzentrationslager Theresienstadt umgebracht wurde. Hierzu gehört auch der verdienstvolle Generalsekretär der Gesellschaft, Max Hirsch, der ihre ersten sieben Kongresse vorbereitete und, solange es ging, internationale Verbindungen aufrecht erhielt, bis er 1933 über die Tschechoslowakei in die Sowjetunion fliehen musste. Bekannt sind auch die Schicksale weiterer jüdischer Mitglieder der Gesellschaft wie Karl Harpuder, Jacques Kroner und August Laqueur sowie die politisch Verfolgten Rudolf Jürgens, Mitherausgeber der Zeitschrift für Rheumabekämpfung, aber auch der erste Präsident unserer Gesellschaft, Eduard
Dietrich (1860-1947), der 1933 wegen seiner aktiven Rolle im Evangelischen Diakonie-Verein aus allen Ehrenämtern entfernt wurde. Dr. Dietrich war schon 1920 Vorsitzender der Balneologischen Gesellschaft geworden, im Jahr 1926 beteiligte er sich an der Gründung der Internationalen Rheumaliga in Pieš zusammen mit Jan van Breemen (1874-1961) und Robert Fortescue Fox (1858-1940).

Hans Reiter (1881-1969) war Präsident des Reichsgesundheitsamtes (19331945) und Mitglied des Sachverständigenbeirats für Bevölkerungs- und Rassenpolitik im Reichsinnenministerium, er galt als eifriger Verfechter nationalsozialistischer Ideen [5, 6, 7, 8, 9]. Nach dem Krieg war er in Nürnberg zwei Jahre inhaftiert, wurde aber letztlich nicht angeklagt oder verurteilt. Dies entbindet ihn aber sicher nicht von einer erheblichen Mitverantwortung für die nationalsozialistischen Verbrechen.

Friedrich Wegener (1907-1990) war SA-Sanitäts-Obersturmbannführer und in den Jahren 1939-1944 als Pathologe im Armee- und Zivildienst in Lodz in Polen in der Nähe des Konzentrationslagers Chelmno tätig. Dies kam erst im Jahre 2006 durch die Publikation von A. Woywodt et al. [10] an die Öffentlichkeit. Die von ihm zu tragende Mitverantwortung wird kontrovers beurteilt. Sie wird von polnischen (A. Grzybowski) und amerikanischen (R. de Remee) Kennern der Materie als gering eingeschätzt (die ent- 
sprechende Korrespondenz liegt dem Vorstand vor).

In beiden Fällen ist die Forderung erhoben worden, die entsprechenden Eponyme abzuschaffen, um diesen historischen Erstautoren nicht unangemessenen Ruhm zuteil werden zu lassen $[7,8$, 9, 11]. Bei der Darstellung von Person und Werk des Hans Reiter in der Zeitschrift für Rheumatologie $[5,6]$ sind die Argumente für und gegen eine solche Vorgehensweise erörtert worden.

Während das Reiter-Syndrom in Deutschland und Europa als seltene Sonderform der ebenfalls nicht mehr häufigen reaktiven Arthritis gewissermaßen im Rahmen des natürlichen Verlaufs nur noch selten erwähnt wird, ist dies in den USA anders, da dort die Diagnose „incomplete Reiter's syndrome“ häufig gestellt wurde [12].

Beim „Morbus Wegener" ist die Situation dadurch wesentlich beeinflusst, dass sich durch die Entdeckung der antineutrophilen zytoplasmatischen Antikörper (ANCA) und ihrer Assoziation zu den Vaskulitiden [13, 14] die pathophysiologischen und diagnostischen Grundlagen der Erkrankung verändert haben.

Angesichts der weit reichenden Konsequenzen einer Änderung in der medizinischen Nomenklatur befürwortet die DGRh als wissenschaftliche Fachgesellschaft, Änderungen nur auf der Grundlage einer sorgfältigen medizinhistorischen Prüfung unter streng wissenschaftlichen transparenten Gesichtspunkten und durch ein autorisiertes internationales Gremium durchzuführen.

Zusammengefasst verurteilt die DGRh die nationalsozialistischen Verbrechen und insbesondere das Verhalten aller Ärzte, die dazu aktiv beigetragen haben. Während auf der Grundlage der heutigen Erkenntnisse die Verantwortung von Hans Reiter in diesem Zusammenhang offensichtlich erscheint, ist eine bedeutende Mitschuld von Friedrich Wegener nicht erwiesen. Die DGRh hält es grundsätzlich für wichtig, dass die deutsche (Medizin-)Geschichte, auch im vergleichsweise kleinen Bereich der Rheumatologie, der Nachwelt zur Mahnung dient und deshalb nicht in Vergessenheit gerät. Der Vorstand der DGRh dankt an dieser Stelle ausdrücklich Herrn Prof. Keitel, der sich in den letzten Jahren um die Aufarbeitung der Geschichte der DGRh sehr verdient gemacht hat.

\section{Korrespondenzadressen}

\section{Prof. Dr. J. Braun}

Rheumazentrum Ruhrgebiet

Landgrafenstr. 15, 44652 Herne

j.braun@rheumazentrum-ruhrgebiet.de

\section{Prof. Dr. E. Genth}

Rheumaklinik und Rheumaforschungsinstitut Burtscheider Markt 24, 52066 Aachen mail@ekkehard-genth.de

\section{Literatur}

1. Reiter H (1916) Über eine bisher unerkannte Spirochäteninfektion (Spirochaetosis arthritica). Dtsch Med Wochenschr 42:1535-1536

2. Wegener F (1936) About generalised septic vascular diseases. Verh Deut Pathol Ges 29:202-210

3. Keitel W, Schmidt KL, Hewelt T, Hüge W (2002) 75 Jahre deutsche Gesellschaft für Rheumatologie - ihre Wurzeln und Gründer. Z Rheumatol 61 (Suppl 1):3-19

4. Zaller R (2003) Hans Reiter and the politics of remembrance. Semin Arthr Rheum 32:237-241

5. Keitel W (2004) Hans Reiter und das okulo-urethrosynoviale Syndrom. 3. Der unbekannte Hans Reiter, Wissenschaftler und NS-Propagandist. Z Rheumatol 63:244-249

6. Keitel W (2004) Hans Reiter und das okulo-urethrosynoviale Syndrom. 4. Nachspiel und Erbe. Z Rheumatol 63:341-345

7. Wallace DJ, Weisman M (2000) Should a war criminal be rewarded with eponymous distinction? The double life of Hans Reiter (1881-1969). J Clin Rheum 6:59-64

8. Wallace DJ, Weisman M (2003) The physician Hans Reiter as prisoner of war in Nuremberg. A contextual review of his interrogations (1945-1947). Semin Arthr Rheum 32:208-230

9. Iglesias-Gammara A, Restrepo JF, Valle R, Matteson EL (2005) A brief history of Stoll-Brodie-FiessingerLeroy syndrome (Reiter's syndrome) and reactive arthritis with a translation of Reiter's original 1916 article into English. Curr Rheumat Rev 1:71-79

10. Woywodt A, Haubitz M, Haller H, Matteson EL (2006) Wegener's granulomatosis Lancet 367:1362-1366

11. Luqmani R, Watts R, Matteson EL, Woywodt A, Merkel PA (2009) Letter to the presidents of ACR and EULAR

12. Arnett FC, McClusky OE, Schacter BZ, Lordon RE(1976) Incomplete Reiter's syndrome: discriminating features and HL-A W27 in diagnosis. Ann Intern Med 84:8-12

13. van der Woude FJ, Rasmussen N, Lobatto $S$ et al (1985) Autoantibodies against neutrophils and monocytes: tool for diagnosis and marker of disease activity in Wegener's granulomatosis. Lancet 1(8426):425-429

14. Lüdemann J, Utecht B, Gross WL (1990) Anti-neutrophil cytoplasm antibodies in Wegener's granulomatosis recognize an elastinolytic enzyme. J Exp Med 171: 357-362

\section{Rheumatologen fordern Stär- kung der Arthroseforschung}

Bislang gibt es kein Medikament und keine chirurgische Therapie, um Arthrose und den damit verbundenen Abbau des Gelenkknorpels zu stoppen. Der Präsident der Deutschen Gesellschaft für Rheumatologie e.V., Professor Dr. med. Wolfgang Rüther, betont, dass die Arthrose keine altersbedingte Verschleißkrankheit ist. Am Anfang der Krankheit stehe häufig eine Schädigung des Gelenkknorpels durch Infektionen, Entzündungen, Unfälle oder auch Stoffwechselstörungen. Ein vielversprechender neuer Ansatz ist die Transplantation von Knorpelzellen oder -gewebe. Eine Wiederherstellung der ursprünglichen Gewebequalität gelingt allerdings noch nicht. Außerdem sei die Therapie nur bei abgegrenzten Knorpelschäden möglich, wie sie zum Beispiel als Unfallfolge auftreten können. Damit käme sie nur für einige Patienten in Frage. Derzeit beschränke sich die Therapie der Arthrose leider im Wesentlichen auf eine Schmerzlinderung und auf den Erhalt der Gelenkbeweglichkeit. Bei fortgeschrittener Erkrankung benötigen viele Menschen dann ein Kunstgelenk. Um auch den vielen anderen Arthrose-Patienten eine Therapie anbieten zu können, müssen die Forschungsaktivitäten weiter verstärkt werden. Es gelte nun, in enger Zusammenarbeit von Rheumatologen, Orthopäden und Naturwissenschaftlern Wege zu finden, in den Prozess des Knorpelabbaus einzugreifen.

Quelle:

Deutsche Gesellschaft für Rheumatologie e.V., www.dgrh.de 